\title{
Blockchain Technology: Rising Trend in Various Applications
}

\author{
Dr. Zalte S. S a ${ }^{1}$, Patil P. N ${ }^{\text {b }}$, Deshmukh S. N ${ }^{\text {b }}$, Patil M. G ${ }^{\text {b }}$, Katkar P. S a \\ ${ }^{a}$ Department of Computer Science, Shivaji University, Kolhapur, India \\ ${ }^{\mathrm{b}}$ Department of Computer Science, Vishwakarma College of ACS, Pune, India
}

\begin{abstract}
In this tech world, size of block chain is exponentially growing in this covid pandemic year which was not predicted. This pandemic environment causes digital transformation drive in various areas, particularly via the utilization of block chain or distributed ledger technology. To enhance and accelerate business process in various organization and industry showed a growing interest to adopt this technology. This paper summarized various block chain applications which are widely used in number of sectors and also focus on some challenges becomes hurdle while adopting block chain technology.
\end{abstract}

Keywords: Blockchain, Challenges, Covid, Distributed Ledger, Bitcoin.

\section{Introduction}

Blockchain is a technology that permits dispensed public ledger through immutable facts in an encrypted and secured way. This ensures a safe transaction that cannot be altered. Blockchain is a decentralized transaction, and data management technology [1]. Latest hyper-linked global, or the "global village", is challenged with the upward thrust of human sickness epidemics and pandemics because of complex interactions among a couple of factors which encompass ecological, environmental, and socioeconomic elements. The enormously infectious severe Acute respiratory Syndrome Corona virus2(SARS-CoV-2), which reasons then oval corona virus disorder (cited here after as COVID-19), has been wreaking havoc around the globe. Number of blockchain solutions had been carried out all through the COVID-19 pandemic. Public health strategists and policymakers explore distinct procedures to lessen the devastating consequence so novel corona virus disorder (COVID-19).

Blockchain generation has emerged as a useful resource that may be utilized in several tactics. Many blockchain technologies had been proposed or implemented during the COVID-19 pandemic [2].

\footnotetext{
${ }_{1}^{1}$ Patil M.G. Dept. of Computer Science, Shri S.M. Mahavidyalaya, Pune.

E-mail: minalpatil8491@gmail.com
} 


\section{Challenges in Block chain technology}

As per our discussion in above paragraph, the block chain technology offers very comfort solutions like transparency, decentralization, integrity, immutability, and security without requiring any centralized trusted authority. However, some challenging issues are to be addressed for various applications.

Energy Consumption: - To validate the transaction for insertion into network Proofof-Work mechanism is used, which requires lot of computational power for the processing of complex mathematical puzzles.

Scalability: - Ability to handle plenty number of nodes at a time is challenging task for this technology. It also involves complication calculation for single transaction which may slow down performance.

Privacy: - As it is open ledger, anyone can view the contents, which might be beneficial in many applications, but in case of sensitive application it becomes liability. Lack of Talent: - Currently, there are few employees to build decentralized block chain. Educating employees to work with block chain will be lengthy process.

Security: - Security is another crucial topic here. We have a tendency to all skills each block chain technology boasts regarding its security. However, like every different technology, block chain conjointly comes with a couple of security loop holes. There are various attacks on the network is one among the protection flaws of the network. While imposing these attacks, hackers will take over the network and exploit it in their method. They will even modify method and prohibit others from making a block.

\section{Pillars of Blockchain Architecture:}

The main four pillars of block chain architecture are shown in Figure 1. The blockchain architecture consists of a few essential ideas like decentralization, digital signature, mining, and data integrity [3].

Decentralization: -Rather than to give preference to one vital authority overpowering others in the environment, blockchain explicitly distributes control among all participants inside the transaction chain.

Digital signature: - Block chain allows a change of transactional price the usage of public keys through the mechanism of unique digital anatomy.

Mining: - In a dispensed device, every person mine and digs deep into the statistics that are then evaluated in line with the cryptographic rules and it additionally recognizes miners for confirmation and verification of the transactions.

Data integrity: - Complicated algorithms and agreement among customers guarantees that transaction information, as soon as agreed upon, can't be tampered with and hence remains unaffected. Information saved on block chain acts as an unmarried version of reality for all parties worried as a result reducing the hazard of fraud [4]. 


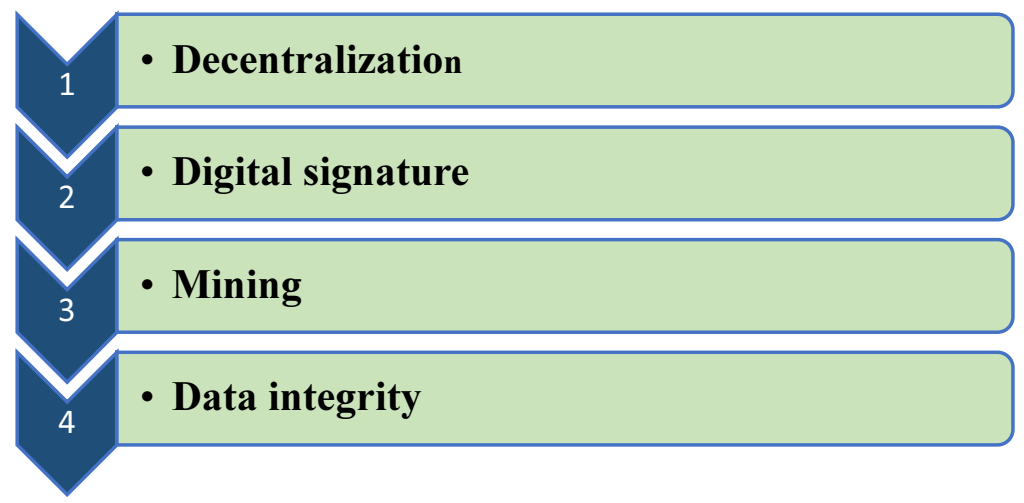

Figure 1. PillarsArchitecture of Blockchain

\section{Block chain Applications:}

Blockchain applications go so much on the far side crypto-currency and bitcoin, with its ability to form a lot of transparency and fairness. Here, we have summarized some applications of blockchain in various sectors as shown in Figure 2.

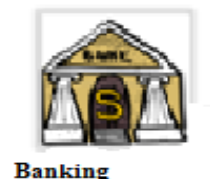

Banking

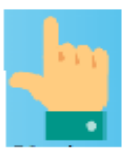

Voting

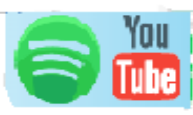

Online Music

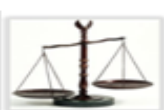

Law Enforcement

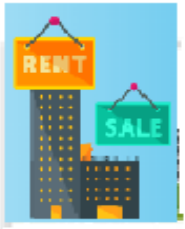

Real Estate

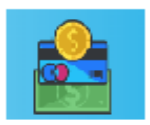

Payments and

Transfer

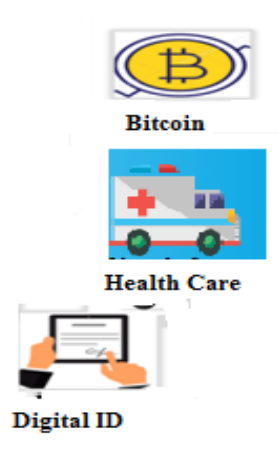

Digital ID

Figure 2. Application of Blockchain

\subsection{Land Registry}

A future-evidence, Block chain the actual estate market is building up new commercial enterprise models of connecting potential shoppers and sellers. In blockchain solution, there's no need of third-party, lawyers and brokers. The ubiquity joined with the real property signs up a workplace in Brazil and advanced the undertaking which pursuits to reduce a fee even as improving, a protection and a transparency. The ubiquity seeks to great immutability to belongings possession data treated through the land an information office. Facts stored on Blockchain are immutable. Block chain-based a totally method to register assets titles could appreciably boom the performance of convincing or even prevent a fraud [5]. 
Merits:- Use of block chain in land registry process can able to transfer land ownership legally to the buyer without involvement of third party. It can also bring transparency and reduce frauds with the help of smart contract. You can generate unique digital id for buyer and seller which accelerate process of ownership transfer. Once registration complete, smart contract update land title for every new buyer and information regarding to this is permanently stored in block chain.

\subsection{Voting:}

Existing voting system faces lot of issues like fraud, long delay time, accessibility, affordability and privacy. Voting strategies stay in many countries a controversial topic, as incident of electoral fraud (invalid or faulty vote, a couple of registration) and the large percentage of abstention frequently shape the end result.

The variation of block chain technology promise trust, security in transaction and transparency. The voters may want to connect with a pc-primarily based system through their laptop, computer or telephone, the use of open-supply code this is open to modify the use of a kind of authentication (biometric, written) prove their identity to this system. Then, they enter their private key to access their proper to vote and the usage of their public key to pick their choice and affirm it. Thus far, three tasks had been founded that sell voting through blockchain systems. The first is, "Bit Congress" that makes use of the Ethereum platform to develop its idea primarily based on the scenario that every voter has get entry to one "vote coin" that enhances him to vote only one time, and his vote will be recorded at the blockchain after the machine verifies it. Normally, the transformation of the voting gadget from paper-primarily based to digital will increase its reliability, and the benefit that gives to the voters [6].

Merits: -The voters can submit their vote from anywhere without burden of geographical barrier and without revealing their original identity. Fake counting of vote can be reduced by counting one ID for one vote. Voter's hash can keep voter's vote confidential. Voter can able to tally whether his/her vote was included or not.

\subsection{Healthcare}

Healthcare systems control and monitor the health of patients with the assist of superior technologies. The advancement of these structures desires to contain an unequivocal spotlight on making these systems efficient [7]. Blockchain generation should play a pivotal position in the healthcare enterprise with numerous programs in areas like public healthcare control, longitudinal healthcare information, automatic fitness claims adjudication, online affected person get entry to, and person oriented medical studies, drug counterfeiting, medical trial, and precision medicine. Especially, blockchain era and using SCs could resolve issues of clinical credibility of findings (lacking data, endpoint switching, facts dredging, and selective guide) in clinical trials as well as problems of patients' knowledgeable consent [8]. Blockchain carried out to the fitness sector can provide new and powerful possibilities to enhance numerous activities related to the prevention and manipulate of pathologies and, therefore, higher medical danger management within the context of a virulent disease emergency consisting of the current one. The sudden appearance and the speedy and out of control diffusion around the world of Corona virus has shown us no longer only the failure of existent healthcare surveillance systems in directly dealing with the public health emergency, however additionally an obvious lack of advanced predictive systems 
based totally on the sharing of scientific records on a large scale, capable of saves you or as a minimum reduce emergencies of such value [9].

Merits: -Automatic update of patient's health information is possible and information can be sharable with other hospitals or organization through sharable private key. Block chain help to securely tracking of pharmaceutical supplies with full of transparency. It also helps in health insurance process to present medical events in timely manner and helps to reduce fraud.

\subsection{Bitcoin: The first block chain}

A bitcoin is a peer to see digital currency that permits online payment from one party to any other without a want for third party. It turned into proposed to remedy the shortcomings of fiat cash and banks. A bitcoin uses blockchain to report the transaction. (Nakamoto, 2008) The bitcoin protocol become brought in 2008, by using pseudonym Satoshi Nakamoto in a white paper named, "Bitcoin: A Peer-to-Peer digital cash gadget". The bitcoin is the primary and the most popular cryptocurrency. The current marketplace capitalization of bitcoin is $\$ 1186.36$ billion (Statista 2021). Blockchain is the era in the back of the bitcoin. Blockchain can be meant as a running system and bitcoin as one of many programs. Blockchain gives disbursed ledger to report bitcoin transactions and has many makes use of beyond bitcoin [10].

Merits: Users can transfer money to anyone in the network without dealing with the bank or government or third party, so transaction fees are also low for international payments. Anonymity is also preserved because bit coin purchases are discrete. It is easily accessible through computer, laptop etc.

\subsection{Banking:}

Block chain technology is a center, underlying era with promising utility potentialities in the banking industry [11]. Banking and technology are very intently related and innovations have modified banking considerably over the time frame. The virtual improvements within the banking sector commenced with the creation of cash that changed the barter machine, and then the sluggish substitute of wax seal with digital signatures. Such innovation that is changing the banking sector globally is Block chain technology (BCT). Blockchain is shared allotted ledger which stores business transaction to an everlasting unbreakable chain which may be viewed through the parties in a transaction. Blockchaingeneration has the ability to disrupt the financial enterprise applications as it affords permanent and tamper evidence recording of transactions in a disbursed network. It could be extensively applied in digital currency, trade finance, KYC and go border remittances, and so on [12].

Merits: Customer's identity verification process is accelerated using block chain and also this verification can reuse for different services to reduce time. Peer-to peer payment transfer is possible without involvement of third trusted authority.

\subsection{Online Music}

Block chain technology is often seen as "the next thing" that can change the structure of different industries. The music industry is no exception in that respect as a lot has been already said regarding the implications of block chain for the industry as a 
whole, including an increase in the value captured by artists, a shift of market power, disintermediation, more transparency and more efficient systems [13].

Block chain technology has stand up as a technique to bring secure, reliable, and scalable distributed transaction processing to music licensing. Significant investment and technical talent have entered the music trade through block chain technology. A block chain is a distributed ledger - a database of transactions, of which each and every party to each group action incorporates a complete copy at all times. Block chains take advantage of low-cost storage, reliable internet connection, and progressive security to switch massive, monolithic, proprietary systems with open, light-weight, distributed systems [14].

Currently, music will be streamed and downloaded at the clicking of a button, however, payments to those that build that music can be slow and opaque. Block chain technology offers transparency through the worth chain, permitting musicians and their managers to examine specifically how much cash they're owed, as against a culture of non -disclosure agreements and black boxes. Though some of the claims created for block chain technology are premature, it seems to possess a minimum of the potential to transform the music business [15].

MEDIACHAIN, UJO, CHOON, OPEN MUSIC INITIATIVE, MUSICOIN and many more music companies started with adopting this methodology to ensure that the artist are paid fairly, their royalty stipulations without the hassle of confusing third parties or contingencies, eliminates the confusion of music ownership and facilitates crowd funding for up-and-coming artists and rewards listeners for creating personalized playlists [16].

Merits: - Intellectual property rights of creative items can be preserved by distributed ledger by keeping evidence of ownership in block chain. Artists also aware of how much money made by publishing music album, so they can received fair amount within time.

\section{Conclusion}

In this era, of the blockchain, although in case of huge scale implementation engross some major serious problems that can't be unmarked. These challenges cause the sluggish adoption of block chain technology by numerous industries and organizations. The acceptance of blockchain's potential to act as a completely unique disruptor, across multiple industries and businesses could presently cause mass adoption. However, blockchain technology is going through a hype phase. To get rid of some hurdles and power the techno world we have to wait. In the future, career opportunities are growing to extend at an alarming rate. Integration with new-age technology like IoT can facilitate in building secure infrastructure. Enterprise block chain can still mature and develop, resulting in high job prospects and sensible pay.

\section{References}

[1]. Yli-Huumo, J., Ko, D., Choi, S., Park, S., \&Smolander, K. (2016). Where is current research on blockchain technology?-a systematic review. PloS one, 11(10), e0163477.

[2]. Abd-alrazaq, A. A., Alajlani, M., Alhuwail, D., Erbad, A., Giannicchi, A., Shah, Z., ... \&Househ, M. (2020). Blockchain technologies to mitigate COVID-19 challenges: A scoping review. Computer Methods and Programs in Biomedicine Update, 100001. 
[3]. Panicker, S., Patil, V., \& Kulkarni, D. (2016). An overview of blockchain architecture and it's applications. Int. J. Innov. Res. Sci., Eng. Technol., 5(11), 20074-20084.

[4]. Shah, T., \& Jani, S. (2018). Applications of blockchain technology in banking \& finance. ParulCUniversity, Vadodara, India.

[5]. Barbieri, M., \&Gassen, D. (2017, March). Blockchain-can this new technology really revolutionize the land registry system. In Responsible Land Governance: Towards an Evidence Based Approach: Proceedings of the Annual World Bank Conference on Land and Poverty (pp. 1-13).

[6]. Foroglou, G., \&Tsilidou, A. L. (2015, May). Further applications of the blockchain. In 12th student conference on managerial science and technology (pp. 1-8)..

[7]. Singh, P., \& Singh, N. (2020). Blockchain With IoT and AI: A Review of Agriculture and Healthcare. International Journal of Applied Evolutionary Computation (IJAEC), 11(4), 13-27.

[8]. Shen, C., \& Pena-Mora, F. (2018). Blockchain for cities - a systematic literature review. Ieee Access, 6, 76787-76819.

[9]. Fusco, A., Dicuonzo, G., Dell'Atti, V., \&Tatullo, M. (2020). Blockchain in healthcare: Insights on COVID-19. International Journal of Environmental Research and Public Health, 17(19), 7167.

[10]. Khadka, R. (2020). The impact of blockchain technology in banking: How can blockchain revolutionize the banking industry?,Thesis in CENTRIA UNIVERSITY OF APPLIED SCIENCES Business Management October 2020.

[11]. Guo, Y., \& Liang, C. (2016). Blockchain application and outlook in the banking industry. Financial Innovation, 2(1), 1-12.

[12]. Gupta, A., \& Gupta, S. (2018). Blockchain Technology: Application in Indian Banking Sector. Delhi Business Review, 19(2), 75-84.

[13]. Sitonio, Camila\&Nucciarelli, Alberto. (2018). The impact of blockchain on the music industry, 29th European Regional ITS Conference, Trento 2018 184968, International Telecommunications Society (ITS).

[14]. Rosenblatt, B. (2017). Watermarking technology and blockchains in the music industry. Retrieved from Digimarc(https://www.digimarc.com/docs/defaultsource/digimarcresources/whitepaper-blockchain-inmusicindustry.pdf.

[15]. De León, I. L., \& Gupta, R. (2017). The impact of digital innovation and blockchain on the music industry. Inter-American Development Bank.(Nov 2017). Available online: https://publications.iadb. org/en/impact-digital-innovation-and-blockchain-music-industry (accessed on 23 June 2020).

[16]. Daley, S. (17). Blockchain Music Companies Reshaping a Troubled Industry. Built In. Retrieved on November, 3, 2019. 